\title{
FURTHER RESULTS FROM THE GALACTIC O-STAR SPECTROSCOPIC SURVEY: RAPIDLY ROTATING LATE ON GIANTS
}

\author{
Nolan R. Walborn ${ }^{1}$, Jesús Maíz Apellániz ${ }^{2,6}$, Alfredo Sota ${ }^{2}$, Emilio J. Alfaro ${ }^{2}$, \\ Nidia I. Morrell ${ }^{3}$, Rodolfo H. Barbá ${ }^{4,6,7}$, Julia I. Arias ${ }^{4}$, And Roberto C. GameN ${ }^{5}$ \\ ${ }^{1}$ Space Telescope Science Institute, 3700 San Martin Drive, Baltimore, MD 21218, USA; walborn@ stsci.edu \\ ${ }^{2}$ Instituto de Astrofísica de Andalucía-CSIC, Glorieta de la Astronomía s/n, 18008 Granada, Spain; jmaiz@iaa.es, sota@iaa.es, emilio@iaa.es \\ ${ }^{3}$ Las Campanas Observatory, Observatories of the Carnegie Institution of Washington, Casilla 601, La Serena, Chile; nmorrell@ @ co.cl \\ ${ }^{4}$ Departamento de Física, Universidad de La Serena, Cisternas 1200 Norte, La Serena, Chile; rbarba@dfuls.cl, julia@dfuls.cl \\ ${ }^{5}$ Instituto de Astrofísica de La Plata-CONICET and Facultad de Ciencias Astronómicas y Geofísicas, Universidad Nacional de La Plata, \\ Paseo del Bosque s/n, 1900 La Plata, Argentina; rgamen@ fcaglp.unlp.edu.ar \\ Received 2011 June 23; accepted 2011 August 26; published 2011 September 28
}

\begin{abstract}
With new data from the Galactic O-Star Spectroscopic Survey, we confirm and expand the ONn category of late-O, nitrogen-enriched $(\mathrm{N})$, rapidly rotating $(\mathrm{n})$ giants. In particular, we have discovered two "clones" (HD 102415 and HD 117490) of one of the most rapidly rotating O stars previously known (HD 191423, "Howarth's Star"). We compare the locations of these objects in the theoretical H-R diagram to those of slowly rotating ON dwarfs and supergiants. All ON giants known to date are rapid rotators, whereas no ON dwarf or supergiant is, but all ON stars are small fractions of their respective spectral-type/luminosity-class/rotational subcategories. The ONn giants, displaying both substantial processed material and high rotation at an intermediate evolutionary stage, may provide significant information about the development of these properties. They may have preserved high initial rotational velocities or may have been spun up by terminal-age main-sequence core contraction; alternatively, and perhaps more likely, they may be products of binary mass transfer. At least some of them are also runaway stars.
\end{abstract}

Key words: stars: abundances - stars: early-type - stars: evolution - stars: fundamental parameters - stars: rotation

\section{INTRODUCTION}

Massive stars burn hydrogen to helium on the CNO cycle(s); the slowest reactions have the effect of leaving nearly all of these elements as nitrogen, at the outset and for their duration. If this partially (since the conversion of $\mathrm{H}$ to He proceeds on the nuclear timescale) processed material is mixed to the surface before the reactions run to completion, enhanced nitrogen-to-carbon and nitrogen-to-oxygen abundance ratios become visible. Thus, direct observations of products from nuclear reactions inside the stars can be made and may provide vital evolutionary diagnostics, if they can be correctly interpreted.

Early on it was realized that Wolf-Rayet (W-R) stars of the nitrogen sequence (WN) are revealing the products of H-burning, while the carbon sequence (WC) is more evolved and shows He-burning products (see Crowther 2007 for a recent review and references). However, the phenomenology (let alone the physics) is highly complex: for instance, very massive stars may become WNs solely through internal mixing and mass loss; at intermediate masses, WN stars may be post-red supergiants; and mass transfer in binary systems can produce WN objects at all masses down to at least $20 M_{\odot}$. More recently, it has been shown that the timescale and degree of these effects are functions of the initial stellar rotational velocity (Maeder \& Meynet 2000; Heger \& Langer 2000).

An analogous dichotomy among certain absorption-line OB spectra was described by Walborn (1971a), who adopted a classification notation analogous to that for W-R spectra, i.e., $\mathrm{OBN}$ and $\mathrm{OBC}$. However, it soon became apparent from

\footnotetext{
6 Visiting Astronomer, Las Campanas Observatory.

7 Also at Instituto de Ciencias Astronómicas de la Tierra y del Espacio (ICATE-CONICET), Avenida España 1512 Sur, J5402DSP, San Juan, Argentina.
}

their strong hydrogen lines and space distribution that the latter category is less evolved than the former, which in turn led to the suggestion that the morphologically normal majority of $\mathrm{OB}$ supergiants may already display some mixing of processed material in their spectra, while the OBN are extreme cases (Walborn 1976). This interpretation has received substantial subsequent support (Smith \& Howarth 1994; Maeder \& Conti 1994).

The OBN/OBC phenomenology is also complex and likely has a range of origins among different objects, just as in the W-R case. Moreover, its detectability is a strong function of the available spectroscopic criteria, which vary as a function of spectral type; the late-O and early-B range is particularly favorable, because of the number of comparably strong $\mathrm{C}, \mathrm{N}$, and $\mathrm{O}$ features present, which are strongest in the supergiants. Examples in high-quality digital data can be found in Walborn \& Fitzpatrick (1990), Walborn \& Howarth (2000), and Walborn (2009a). Quantitative measurements and analysis can mitigate these selection effects to a considerable extent. Recent investigations from different perspectives have been presented by Fraser et al. (2010), Przybilla et al. (2010), and Lyubimkov et al. (2010). Rotation is again a key parameter, as is the possibility of angular momentum and abundance alterations by mass transfer in binary systems (Langer et al. 2008). These effects may produce systematic differences in mixing among some clusters and associations.

Howarth \& Smith (2001) showed that the remarkable late-O giant HD 191423 ("Howarth's Star") is one of the two most rapidly rotating massive stars analyzed so far and displays a strong nitrogen enhancement in its spectrum; thus, it combines two of the key parameters of interest in the present context. Walborn (2003) included it in a small, newly defined category of ONn spectra, where the " $\mathrm{N}$ " denotes nitrogen enhancement while "n" (for "nebulous") is the classical descriptor of broadened absorption lines. Here we report the 
discovery of two extremely similar spectra and improve the spectral types of several other members of the ONn category, thus expanding its membership and confirming its existence. We also further discuss the characteristics and possible significance of this category.

\section{OBSERVATIONS}

The Galactic O-Star Spectroscopic Survey (GOSSS; Maíz Apellániz et al. 2010), including its data and analysis procedures, is extensively discussed by Sota et al. (2011), which will not be repeated in detail here. Briefly, GOSSS is a systematic, uniform survey of the blue-violet spectra of all accessible Galactic O stars, with high signal-to-noise ratio (S/N, 200-300) digital data covering at least 3900-5000 $\AA$ at moderate resolution $(R \sim 2500)$. As discussed in more detail below, this work has improved the definition of the spectral-classification system and it is revealing numerous objects and categories of special interest (e.g., Walborn et al. 2010). The present results constitute another example of the latter. Several objects discussed here pertain to future GOSSS installments covering the southern hemisphere.

\section{RESULTS}

A key spectral segment in the full current membership of the ONn class is presented in Figure 1, with the prototype subgiant ON spectrum of HD 201345 for comparison (Walborn 1970; its spectral type has been refined from the original in the GOSSS data). The defining ON morphology of $\mathrm{N}$ III $\lambda \lambda 4634,4640-4642$ and the C III $\lambda 4650$ blend, with the $\mathrm{N}$ III $\lambda \lambda 4640-4642$ blend dominant, is clearly seen. As shown by the further comparison object HD 96264, an O9.5 III standard, in morphologically normal spectra the $\mathrm{C}$ III feature is far stronger than the N III. (In OC spectra it is even more so.)

It can also be readily seen that the absorption lines of all the class III and II objects are appreciably broadened to various degrees, and the progressive effects on the N III/C III blends are clear. Howarth \& Smith (2001) showed how the spectrum of HD 191423, including the CNO anomalies, is perfectly matched by a rotationally broadened version of that of HD 201345. They derived $v \sin i=435 \mathrm{~km} \mathrm{~s}^{-1}$ for HD 191423, the largest value found for an $\mathrm{O}$ star. ${ }^{8}$ In the rotational line-broadening measures calculated by the digital classifier developed by J.M.A. for GOSSS, the descriptors introduced by Walborn (1971b) have the following correspondences: (n) to $200, \mathrm{n}$ to 300 , and $\mathrm{nn}$ to $400 \mathrm{~km} \mathrm{~s}^{-1}$, in excellent agreement with the directly determined value for HD 191423, as well as those for several other objects in the figure listed by Walborn (2003). Figure 1 shows the remarkable similarity between the previously undiscussed spectra of the southern stars HD 102415 and HD 117490, and that of HD 191423, including the extreme line broadening.

As discussed in more detail by Sota et al. (2011), the quality and size of the GOSSS data set have motivated some refinements in the spectral classification system and revisions to the spectral types of a number of individual stars. These developments affect the ONn sample in the following specific ways. (1) The definitions of types 08.5 , O9, and 09.5 have been standardized at all luminosity classes in terms of the line ratios $\mathrm{He}_{\mathrm{I}} \lambda 4144 / \mathrm{He}$ II $\lambda 4200$ and $\mathrm{He}_{\mathrm{I}} \lambda 4387 / \mathrm{He}_{\text {II }} \lambda 4541$, which both have values of unity at $\mathrm{O} 9$ and deviate from

\footnotetext{
8 Herrero et al. (1992) derived $450 \mathrm{~km} \mathrm{~s}^{-1}$ for this same star; see also Villamariz et al. (2002) further discussed below.
}

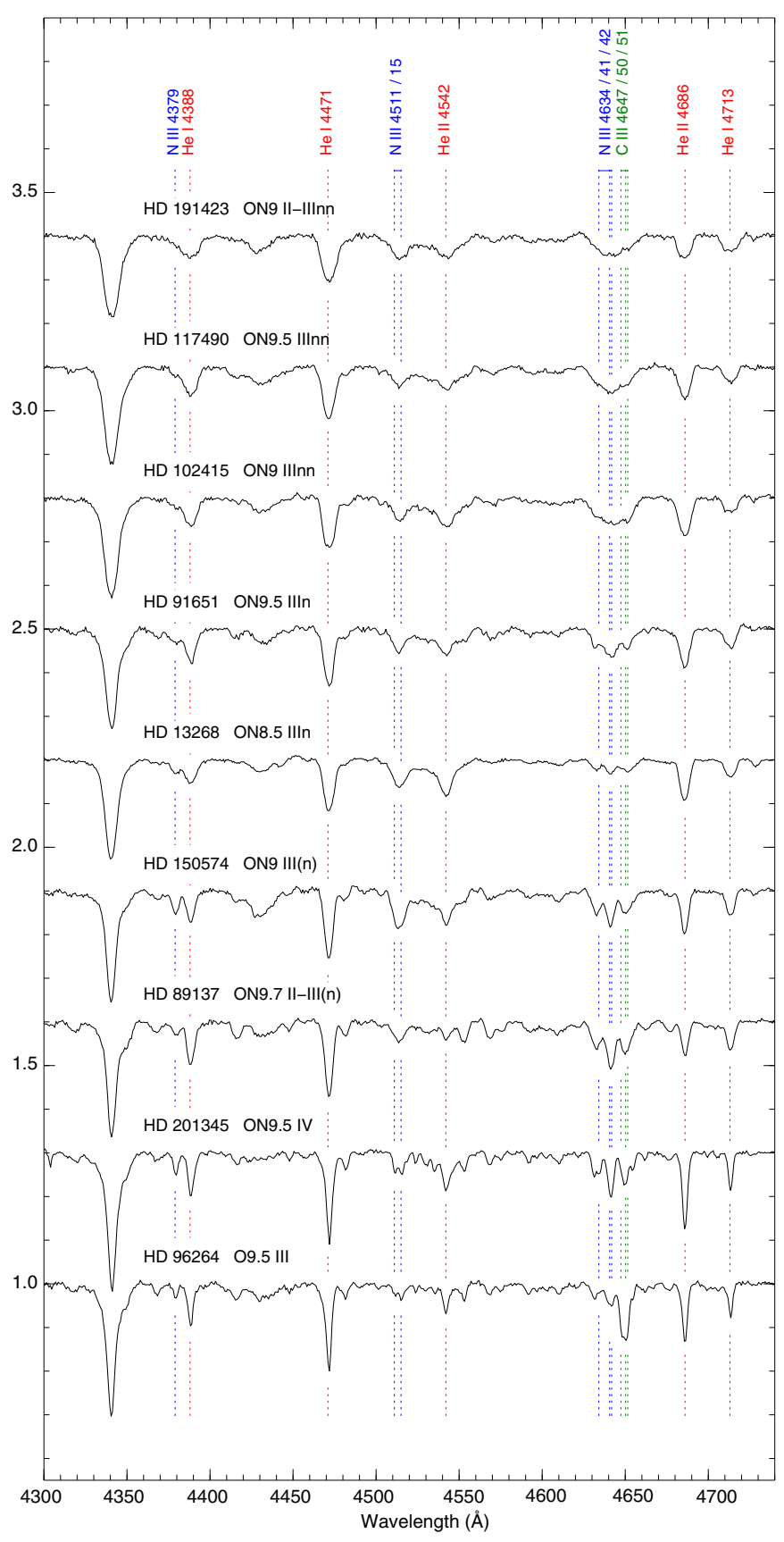

Figure 1. $v \sin i$ sequence of ONn giant spectra in the blue-green region, with the ON subgiant HD 201345 and the normal standard HD 96264 for comparison. The ordinate is rectified continuum intensity.

that in opposite senses to either side. (2) The O9.7 spectral type, based upon a value near unity of the ratio He II $\lambda 4541 /$ Si III $\lambda 4552$, is now used uniformly in all luminosity classes; previously it had been defined only for classes I and II. That revision has been implemented by shifting some B0 and B0.2 types of classes III-V, including standards, to the next earlier type; thus the consistency of the horizontal types has been improved across the vertical classes. (3) The ON defining morphology in the adjacent $\mathrm{N}_{\text {III }} \lambda 4640$ and C III $\lambda 4650$ blends was often difficult to discern with confidence in rapid rotators on photographic spectrograms because of the low contrast. The high-S/N digital data improve this definition substantially, allowing several previously suspected but uncertain cases to be confirmed here; some historical notes for individual stars 
Table 1

Observational and Physical Parameters for ON, OB, and OBC Stars

\begin{tabular}{|c|c|c|c|c|c|c|c|c|}
\hline Name & R.A. (2000.0) & Decl. (2000.0) & Spectral Type & $V$ & $B-V$ & $M_{V}$ & $T_{\text {eff }}$ & $\mathrm{BC}$ \\
\hline \multicolumn{9}{|c|}{ Supergiants } \\
\hline HD 123008 & $14: 07: 30.650$ & $-64: 28: 08.82$ & ON9.5 Iab & 8.837 & +0.371 & -6.5 & 30500 & -2.9 \\
\hline HD 191781 & 20:09:50.581 & $+45: 24: 10.44$ & ON9.7 Iab & 9.533 & +0.636 & -6.5 & 30050 & -2.8 \\
\hline HD 122879 & $14: 06: 25.157$ & $-59: 42: 57.25$ & B0 Ia & 6.410 & +0.124 & -7.0 & 29600 & -2.8 \\
\hline HD 148546 & $16: 30: 23.312$ & $-37: 58: 21.15$ & O9 Iab & 7.711 & +0.291 & -6.5 & 31400 & -3.0 \\
\hline HD 149038 & $16: 34: 05.023$ & $-44: 02: 43.14$ & O9.7 Iab & 4.910 & +0.078 & -6.5 & 30050 & -2.8 \\
\hline HD 154368 & $17: 06: 28.371$ & $-35: 27: 03.76$ & O9.5 Iab & 6.133 & +0.509 & -6.5 & 30500 & -2.9 \\
\hline HD 188209 & 19:51:59.068 & $+47: 01: 38.44$ & O9.5 Iab & 5.625 & -0.064 & -6.5 & 30500 & -2.9 \\
\hline HD 195592 & $20: 30: 34.970$ & $+44: 18: 54.87$ & O9.7 Ia & 7.080 & +0.870 & -7.0 & 30050 & -2.8 \\
\hline HD 202124 & $21: 12: 28.389$ & $+44: 31: 54.14$ & O9 Iab & 7.813 & +0.230 & -6.5 & 31400 & -3.0 \\
\hline HD 104565 & 12:02:27.795 & $-58: 14: 34.36$ & OC9.7 Iab & 9.265 & +0.363 & -6.5 & 30050 & -2.8 \\
\hline HD 152249 & $16: 54: 11.641$ & $-41: 50: 57.27$ & OC9 Iab & 6.463 & +0.202 & -6.5 & 31400 & -3.0 \\
\hline HD 152424 & $16: 55: 03.331$ & $-42: 05: 27.00$ & OC9.5/9.7 Ia & 6.311 & +0.400 & -7.0 & 30500 & -2.9 \\
\hline HD 154811 & 17:09:53.086 & $-47: 01: 53.19$ & OC9.7 Iab & 6.921 & +0.394 & -6.5 & 30050 & -2.8 \\
\hline HD 194280 & $20: 23: 26.313$ & $+38: 56: 21.01$ & BC0 Iab & 8.390 & +0.760 & -6.5 & 29600 & -2.8 \\
\hline \multicolumn{9}{|c|}{ Giants } \\
\hline HD 13268 & 02:11:29.700 & $+56: 09: 31.70$ & ON8.5 IIIn & 8.182 & +0.128 & -5.6 & 32900 & -3.1 \\
\hline HD 89137 & $10: 15: 40.086$ & $-51: 15: 24.08$ & ON9.7 II-III(n) & 7.974 & -0.045 & -5.5 & 30250 & -2.8 \\
\hline HD 91651 & $10: 33: 30.301$ & $-60: 07: 40.04$ & ON9.5 IIIn & 8.849 & -0.008 & -5.3 & 30800 & -2.9 \\
\hline HD 102415 & $11: 46: 54.404$ & $-61: 27: 46.99$ & ON9 IIInn & 9.146 & +0.120 & -5.6 & 31800 & -3.0 \\
\hline HD 117490 & $13: 32: 08.600$ & $-60: 48: 55.47$ & ON9.5 IIInn & 8.902 & +0.033 & -5.3 & 30800 & -2.9 \\
\hline HD 150574 & 16:44:07.209 & $-46: 08: 29.85$ & ON9 III(n) & 8.497 & +0.232 & -5.6 & 31800 & -3.0 \\
\hline HD 191423 & 20:08:07.113 & $+42: 36: 21.98$ & ON9 II-IIInn & 8.030 & +0.160 & -5.75 & 31700 & -3.0 \\
\hline HD 10125 & $01: 40: 52.762$ & $+64: 10: 23.13$ & O9.7 II & 8.220 & +0.310 & -5.9 & 30200 & -2.8 \\
\hline HD 15137 & $02: 27: 59.811$ & $+52: 32: 57.60$ & O9.5 II-IIIn & 7.870 & +0.030 & -5.6 & 30650 & -2.9 \\
\hline HD 24431 & $03: 55: 38.420$ & $+52: 38: 28.75$ & O9 III & 6.732 & +0.371 & -5.6 & 31800 & -3.0 \\
\hline HD 93521 & $10: 48: 23.511$ & $+37: 34: 13.09$ & O9.5 IIInn & 7.040 & -0.280 & -5.3 & 30800 & -2.9 \\
\hline HD 96264 & 11:04:55.501 & $-61: 03: 05.79$ & O9.5 III & 7.606 & -0.064 & -5.3 & 30800 & -2.9 \\
\hline HD 114737 & $13: 13: 45.528$ & $-63: 35: 11.75$ & O8.5 III & 7.995 & +0.172 & -5.6 & 32900 & -3.1 \\
\hline HD 154643 & 17:08:13.983 & $-35: 00: 15.68$ & O9.7 III & 7.165 & +0.278 & -5.1 & 30300 & -2.8 \\
\hline HD 189957 & 20:01:00.005 & $+42: 00: 30.83$ & O9.7 III & 7.806 & +0.013 & -5.1 & 30300 & -2.8 \\
\hline HD 207198 & $21: 44: 53.278$ & $+62: 27: 38.05$ & O9 II & 5.943 & +0.311 & -5.9 & 31600 & -3.0 \\
\hline \multicolumn{9}{|c|}{ Dwarfs } \\
\hline HD 12323 & $02: 02: 30.126$ & $+55: 37: 26.38$ & ON9.5 V & 8.900 & -0.092 & -4.1 & 31900 & -3.0 \\
\hline HD 14633 & $02: 22: 54.293$ & $+41: 28: 47.72$ & ON8.5 V & 7.458 & -0.212 & -4.4 & 33900 & -3.2 \\
\hline HD 48279 & $06: 42: 40.548$ & $+01: 42: 58.23$ & ON8.5 V & 7.910 & +0.136 & -4.4 & 33900 & -3.2 \\
\hline HD 201345 & $21: 07: 55.416$ & $+33: 23: 49.25$ & ON9.5 IV & 7.660 & -0.130 & -4.7 & 31350 & -3.0 \\
\hline HD 46149 & $06: 31: 52.533$ & $+05: 01: 59.19$ & $\mathrm{O} 8.5 \mathrm{~V}$ & 7.601 & +0.171 & -4.4 & 33900 & -3.2 \\
\hline HD 46202 & $06: 32: 10.471$ & $+04: 57: 59.79$ & $09.5 \mathrm{~V}$ & 8.182 & +0.177 & -4.1 & 31900 & -3.0 \\
\hline HD 93028 & $10: 43: 15.340$ & $-60: 12: 04.21$ & O9 IV & 8.361 & -0.071 & -5.0 & 32350 & -3.0 \\
\hline HD 93027 & $10: 43: 17.954$ & $-60: 08: 03.29$ & O9.5 IV & 8.720 & -0.020 & -4.7 & 31350 & -3.0 \\
\hline HD 149757 & $16: 37: 09.530$ & $-10: 34: 01.75$ & O9.5 IVnn & 2.565 & +0.019 & -4.7 & 31350 & -3.0 \\
\hline HD 214680 & $22: 39: 15.679$ & $+39: 03: 01.01$ & O9 V & 4.879 & -0.201 & -4.3 & 32900 & -3.1 \\
\hline
\end{tabular}

are given below. (4) The same uncertainty affected luminosity classes based primarily upon He II $\lambda 4686 / \mathrm{He}$ I $\lambda 4713$ in rapid rotators; it is greatly alleviated in the digital data, which has led to some other revisions.

The digital classifier used with the GOSSS data further contributes to improving the systematic and random reliability of the results, because it compensates for the tendency of the eye to be drawn to central depths rather than equivalent widths in digital data. That of course can lead to systematic effects as a function of line broadening or resolution. The classifier displays any sequence of standard spectra consecutively, either overplotted or displaced from the unknown on the screen. Moreover, the line widths (and consequently, depths) in the standards can be adjusted and matched to those of the unknown in terms of the $\mathrm{n}$ parameter discussed above. The program actually computes composite Doppler profiles for a rotating disk. Second-order effects such as limb darkening, oblateness, or non-rotational broadening are not included, as appropriate for the relatively low resolution of the GOSSS data. High-resolution spectroscopic data for as many of these stars as feasible are being obtained in the context of the associated OWN (Barbá et al. 2010; Arias et al. 2010) and IACOB (Simón-Díaz et al. 2011) programs and will receive full quantitative analyses.

Some parameters of the ONn stars, together with a substantial number of other late $\mathrm{ON}$, normal $\mathrm{O}$, and $\mathrm{OC}$ objects, are listed in Table 1. All of the original ON/OC stars from Walborn (1976) are included here, together with morphologically normal comparison spectra of the same two-dimensional types, as an optimum sample for future quantitative analysis. Note particularly three very rapidly rotating giants/subgiants without nitrogen enhancement (although two of them have enhanced helium; Howarth \& Smith 2001), all of which are also runaway stars (see Section 4.2 below). It will be of some interest to understand their evolutionary histories in contrast to those of the 
Table 2

Velocities, Galactic Latitudes, and Nitrogen Abundances for ON Stars

\begin{tabular}{|c|c|c|c|c|c|c|c|}
\hline Name & Spectral Type & $\begin{array}{c}v \sin i^{\mathrm{b}} \\
\left(\mathrm{km} \mathrm{s}^{-1}\right)\end{array}$ & $\begin{array}{c}v_{r} \\
\left(\mathrm{~km} \mathrm{~s}^{-1}\right)\end{array}$ & Source ${ }^{c}$ & $\begin{array}{c}b \\
\text { (deg) }\end{array}$ & $\mathrm{N}^{\mathrm{d}}$ & Source $^{\mathrm{e}}$ \\
\hline \multicolumn{8}{|c|}{ Supergiants } \\
\hline HD 123008 & ON9.5 Iab & 98 & $-21,-36 \mathrm{C}$ & 1,2 & -2.8 & $\geqslant 1.8$ & 1 \\
\hline HD 191781 & ON9.7 Iab & $\sim 100$ & $-13,-10 \mathrm{~V} ?$ & 3,4 & +6.6 & $\cdots$ & $\cdots$ \\
\hline \multicolumn{8}{|c|}{ Giants } \\
\hline HD 13268 & ON8.5 IIIn & 309 & $-127,-123 \mathrm{C}$ & 5,6 & -5.0 & $\cdots$ & $\ldots$ \\
\hline HD 89137 & ON9.7 II-III(n) & 202 & $+3,-25 \mathrm{~V} ?$ & 1,2 & +4.4 & 25 & 2 \\
\hline HD 91651 & ON9.5 IIIn & 292 & $-41 \mathrm{SB} 2$ & 1,2 & -1.7 & $\ldots$ & $\ldots$ \\
\hline HD 102415 & ON9 IIInn & $\sim 400$ & $-10 \mathrm{~V} ?$ & 7 & +0.4 & $\cdots$ & $\cdots$ \\
\hline HD 117490 & ON9.5 IIInn & $\sim 400$ & +3 SB2? & 7 & +1.7 & $\cdots$ & $\cdots$ \\
\hline HD 150574 & ON9 III(n) & $\sim 200$ & $-56 \mathrm{SB} 2$ & 2 & -0.2 & $\ldots$ & $\ldots$ \\
\hline HD 191423 & ON9 II-IIInn & 435 & $-38,-90 \mathrm{~V} ?$ & 3,8 & +5.4 & 3.1 & 3 \\
\hline \multicolumn{8}{|c|}{ Dwarfs } \\
\hline HD 12323 & ON9.5 V & 131 & $-42 \mathrm{SB}$ & 4,6 & -5.9 & $\geqslant 2.8$ & 1 \\
\hline HD 14633 & ON8.5 V & 134 & $-38 \mathrm{SB} 1$ & 9 & -18.2 & $\geqslant 2.8,13$ & 1,2 \\
\hline HD 48279 & ON8.5 V & 128 & $+29 \mathrm{C}$ & 2,4 & -1.2 & $\geqslant 2.0,16$ & 1,2 \\
\hline HD 201345 & ON9.5 IV & 91 & +20 SB2? & 4 & -9.5 & $\begin{array}{c}33 / 6.5,{ }^{\mathrm{f}} \\
\geqslant 2.0,8\end{array}$ & $\begin{array}{l}4, \\
1,5\end{array}$ \\
\hline
\end{tabular}

Notes.

${ }^{a}$ HD 123008: revised from ON9.7 to ON9.5 in southern GOSSS in prep., but foreseen by Walborn \& Fitzpatrick (1990). HD 13268: ON discovered by Mathys (1989). HD 89137: pec. in Walborn (1976, 2003) and N str. in Garrison et al. (1977); ON confirmed by GOSSS. HD 91651: N str. in Garrison et al. (1977); ON confirmed by GOSSS. HD 102415, 117490: ON discovered by southern GOSSS. HD 191423: ON discovered by Howarth \& Smith (2001). HD 12323, 201345: revised from ON9 to ON9.5 and latter from V to IV in GOSSS. HD 14633, 48279: revised from $\mathrm{O}(\mathrm{N}) 8$ to $\mathrm{O}(\mathrm{N}) 8.5$ in GOSSS and latter from N str. (Walborn 1976) to ON in H. Sana et al. (2011, in preparation).

${ }^{b}$ Projected rotational velocities from Howarth et al. (1997) or Howarth \& Smith (2001) as available; otherwise inferred approximately from the classification line-broadening parameter. Note the perfect agreement with measured values. The actual rotational velocities may be lower if additional line-broadening mechanisms are significant (Simón-Díaz \& Herrero 2007).

c (1) Feast et al. 1963; (2) Levato et al. 1988; (3) Petrie \& Pearce 1961; (4) Bolton \& Rogers 1978; (5) Abt et al. 1972; (6) Kendall et al. 1995; (7) R. Barbá et al. 2011, in preparation; (8) Howarth et al. 1997; (9) Boyajian et al. 2005. $\gamma$ for SB; C: constant; V: variable.

d Nitrogen abundance factors relative to $10 \mathrm{Lac}$, except Source 1 to "normal stars."

e (1) Wollaert et al. 1988; (2) Schönberner et al. 1988; (3) Villamariz et al. 2002; (4) Lester 1973; (5) Schönberner et al. 1984.

${ }^{\mathrm{f}}$ First Source 4 value is an average of nine lines ranging over two orders of magnitude; second value omits four anomalous, excessive lines.

ONn stars. Many of these comparison spectral types have also been refined or newly derived in GOSSS, analogously to the discussion of the ONn class above. The absolute visual magnitudes are from Walborn (1973), while the effective temperatures and bolometric corrections (albeit for nonrotating stars) are from Martins et al. (2005); values for spectral types O9.7-B0 and luminosity class IV have been extrapolated or interpolated, respectively. Projected rotational velocities for the ON sample are given in Table 2, along with radial velocities, galactic latitudes, nitrogen abundance determinations as available, and notes on previous spectral classifications. Two additional Galactic ON supergiants are excluded here: HD 105056, which may be a low-mass (PAGB?) object (Walborn et al. 1980) and BD $+36^{\circ}$ 4063, which is a short-period, spectrum-variable interacting binary (Howarth 2008; Williams et al. 2009).

\section{DISCUSSION}

\subsection{Binarity}

HD 12323, HD 14633, and HD 201345 have been reported as definite or probable spectroscopic binaries by Lester (1973), Bolton \& Rogers (1978), and/or Boyajian et al. (2005). HD 48279 was found to have constant radial velocity by Bolton \& Rogers (1978), Levato et al. (1988), and Mahy et al. (2009). Indeed, binary mass transfer would appear the most likely origin of dwarf or subgiant ON stars. It could also be a possible explanation of the $\mathrm{ONn}$ class in principle (Langer et al.
2008). However, their binary status is currently unclear. Levato et al. reported HD 89137 as probably variable, and HD 91651 and HD 150574 as probably SB2, the last based on the report of double lines by Garrison et al. (1977). HD 13268 was found to have a constant radial velocity by Abt et al. (1972), but see below. A single epoch with double lines was reported for HD 191423 by Petrie \& Pearce (1961), which definitely requires observational follow-up. The new ONn stars HD 102415 and HD 117490 are likely velocity variables. These results are summarized in Table 2, which shows that 10 of the $13 \mathrm{ON}$ stars listed are definite or possible spectroscopic binaries.

Several high-resolution radial-velocity programs on the $\mathrm{O}$ stars are in progress, and the ONn class is an outstanding candidate for further data. The preliminary results in Table 2 for the new ONn stars are from the extensive OWN Survey led by R.C.G and R.H.B. (Barbá et al. 2010; Arias et al. 2010). Of course, given the high incidence of multiplicity among massive stars, the mere fact of binarity does not prove that mass transfer has taken place. A further complication is that some binaries may merge, producing a single, rapidly rotating object.

\subsection{Space Distribution and Motions}

None of the ON or ONn stars listed in Tables 1 and 2 is a definite cluster or association member. On the contrary, as given in Table 2, many of them have unusually high galactic latitudes for OB stars and several have high radial velocities, as previously 


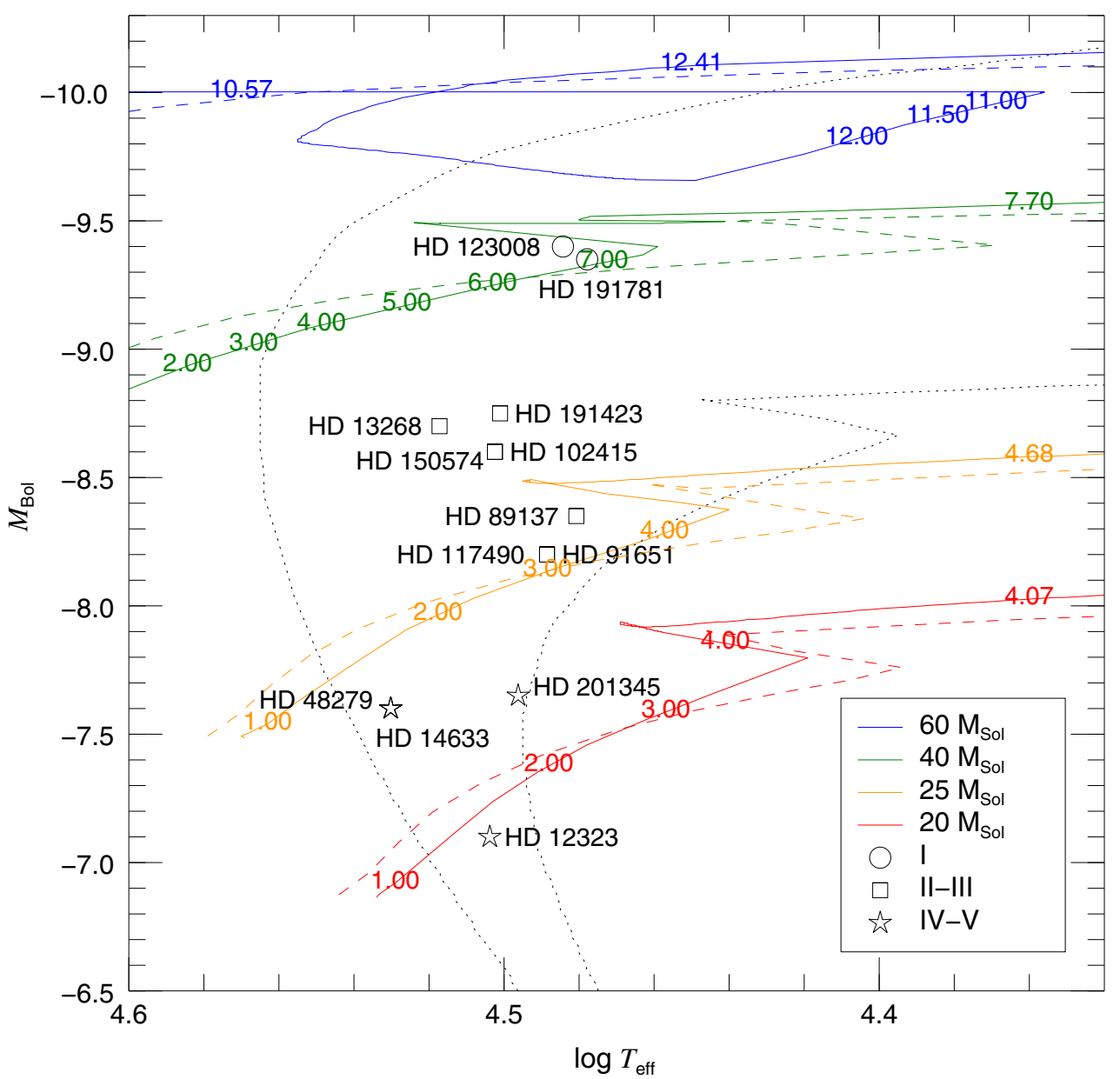

Figure 2. H-R diagram for the ON stars. The ONn stars are the open squares (luminosity classes III-II). Note that the locations of stars with identical two-dimensional classifications are degenerate and shown as single points. The dashed tracks are for nonrotating and the solid tracks for rotating models; the latter are labeled with surface $\mathrm{N}$ abundances relative to the ZAMS value. The dotted lines are nonrotating isochrones for 3.2 and 5.6 Myr.

discussed by Walborn (1970), Bolton \& Rogers (1978), Kendall et al. (1995), Howarth et al. (1997), and Boyajian et al. (2005).

The extreme radial velocity of HD 13268 was found by Abt et al. (1972), its ON nature by Mathys (1989), and both were confirmed and further discussed by Kendall et al. (1995). Abt et al. and Kendall et al. reasonably suggested that HD 13268 is a runaway star, as has been proposed for other ON stars in the references cited in the previous paragraph. Indeed, its position and proper motions are quite similar to those of HD 14633 and HD 15137, for both of which an origin in NGC 654 was suggested by Boyajian et al. HD 12323 also bears comparison in these properties. Clearly this is an important consideration with regard to the origin of these objects, as further discussed below.

\subsection{Abundances}

Unfortunately, quantitative $\mathrm{CNO}$ abundance determinations for the ON stars are sparse and dated. Relative nitrogen abundances as available from the literature are given in Table 2, for reference in the discussion below. However, only that for HD 191423 (Villamariz et al. 2002) has been derived with current techniques. Clearly, state-of-the-art abundance studies of all these stars are essential for substantive progress in their interpretation. The helium abundances should also be investigated systematically. Again, Table 1 here provides an optimum sample for comparative analysis of the relevant categories.

\subsection{Evolution}

\subsubsection{Masses, Ages, and N Enhancements}

An H-R diagram for the ON stars is presented in Figure 2. It displays evolutionary tracks with solar composition for nonrotating (Schaller et al. 1992) and $300 \mathrm{~km} \mathrm{~s}^{-1}$ initially rotating (Meynet \& Maeder 2000) models of 20, 25, 40, and $60 M_{\odot}$. The rotating tracks are labeled with nitrogen abundances relative to the ZAMS. Nonrotating isochrones for 3.2 and 5.6 Myr are also shown. Detailed comparisons of spectraltype calibrations on these tracks were discussed by Walborn \& Lennon (2003). The differences between the rotating and nonrotating cases are relatively small in the domain of the ONn stars, as also seen here, but significant mixing of $\mathrm{N}$ is not predicted by the nonrotating models at these stages.

The stars have been plotted in Figure 2 based on the spectraltype calibrations in Table 1. By that procedure, any star with a given two-dimensional classification appears at the same point in the diagram, regardless of $\mathrm{N}$ or rotational differences. Thus, it is not possible to investigate any systematic effects of those parameters in this way. Also, should the ON stars be products of binary mass transfer, the calibrations for normal stars may not be accurate for them. Reliable individual quantitative analyses and/or distances, which do not exist for most of these stars at 
present but will in the future, are required for more definitive results and comparisons. Although the normal and OC comparison stars are hence not discussed further here, they are retained in the table as optimal comparisons for the ON stars in future work.

According to the HRD, the ONn stars have masses in the range of $25-30 M_{\odot}$ and ages of 4-5 Myr. The ON dwarfs and subgiant are in the $20-25 M_{\odot}$ range, while the two ON supergiants lie on the $40 M_{\odot}$ track. Thus, these subcategories are not evolutionarily related; rather they all have similar spectral types and effective temperatures at least partly as a consequence of the selective detectability of the CNO anomalies at these types. Quantitative work will be required to identify their hotter progenitors, which should have main-sequence spectral types in the O7-O8 range. HD 110360 has a very clear ON7 Vz type, as originally discovered by Mathys (1989) and confirmed by further GOSSS work in progress, in the course of which the "z," possibly related to extreme youth (Walborn 2009b) has been added; however, it has very sharp lines and thus likely a low rotational velocity. Some descendant analogues of the ONn stars may be found among the BN class (Walborn 1976).

Then, one would like to compare the observed nitrogen enhancements (Table 2) with those predicted by the models. Unfortunately, only the observational result for HD 191423 is likely to be sufficiently reliable for a meaningful comparison. Its observed value is in excellent agreement with the prediction for its HRD location, supporting an internal origin of its current $\mathrm{N}$ abundance, as was already concluded by Villamariz et al. (2002). From the strong spectroscopic similarities, it is reasonable to expect that similar results will obtain for the other ONn stars. On the other hand, as noted above, a possible binary nature of HD 191423 requires further investigation, both to confirm it or otherwise and to elucidate any relationship to the $\mathrm{N}$ enhancement if confirmed, and similarly for the other ONn objects.

\subsubsection{Origins}

Several alternative interpretations of the ONn stars may be considered in principle and compared with the available observational and theoretical constraints.

1. Stars with rapid initial rotations are expected to undergo more substantial mixing of processed material to their surfaces (Maeder \& Meynet 2000; Heger \& Langer 2000). In extreme cases they may evolve homogeneously back toward the zero-age main sequence (Langer 1992; Meynet $\&$ Maeder 2000). However, the question of braking by the stellar winds then arises: are the current rotational velocities of the ONn stars compatible with even higher initial values and the expected wind braking? Unfortunately, the massloss rates of late-O dwarfs are currently highly uncertain, with models predicting larger values than implied by observations (Marcolino et al. 2009), so a definitive answer to this question is not at hand.

2. Core contraction at the terminal-age main-sequence stage may increase the rotational velocities, and the ONn stars are near or at that stage. However, so are the far more numerous normal stars of the same spectral types, which argues against that mechanism.

3. Mass transfer in a binary system can produce both rotational acceleration and processed material on the surface of the secondary, whether from the primary or from induced mixing in the secondary (Langer et al. 2008). In extreme cases, a merger may be the end result. In either event, homogeneous evolution may then ensue. If the original primary has already undergone a supernova explosion, the secondary or even the remnant binary may acquire a high space velocity, which is a property of at least some ON stars as discussed above. Blaauw (1993) summarized evidence for helium enhancements in some other runaway stars in this context. High helium abundances in several ON stars have been derived by Schönberner et al. (1988), Herrero et al. (1992), Smith \& Howarth (1994), Kendall et al. (1995), and Howarth \& Smith (2001). In this case, the discussion of single-star evolution becomes irrelevant, which could also be compatible with the rarity of the ON stars.

It is difficult to choose among these alternative interpretations at the present time, except for the likely elimination of (2). However, observational and theoretical developments currently underway may be expected to improve this situation in the relatively near future.

\section{SUMMARY}

The ONn category of nitrogen-rich, rapidly rotating late-O giants has been expanded and enhanced with improved data from the GOSSS. In particular, two southern-hemisphere "clones" (HD 102415 and HD 117490) of the most rapidly rotating ON star previously known (HD 191423) have been added to the category. The properties of the class as currently available have been discussed, including rotation, binarity, space distribution and motions, and nitrogen abundances. A comparison with rotating stellar models has been made. However, the observational data are fragmentary and in some cases inadequate, with the result that alternative interpretations of the ONn class in terms of single-star or binary evolution cannot be definitively distinguished, although the latter may be somewhat favored. This sample of stars, including an extensive list of normal and OC comparison objects, provides an outstanding project for further observations and analysis, some of which are already in progress.

N.R.W. thanks the Sociedad Española de Astronomía for generous international travel and subsistence support, and the Instituto de Astrofísica de Andalucía and its staff for kind hospitality and subsistence (through Spanish Government grants AYA200764052 and AYA2010-17631), during 2010 September-October. Ancillary support, including publication charges, was provided by NASA through grants GO-10898.01 and GO-12179.01 from STScI, which is operated by AURA, Inc., under NASA contract NAS5-26555. J.M.A, A.S., and E.J.A. acknowledge support by the Spanish Government Ministerio de Ciencia e Innovación grants AYA2007-64052 and AYA2010-17631, and by the Junta de Andalucía grant P08-TIC-4075; J.M.A. was also supported by the Ramón y Cajal Fellowship program, cofinanced by the European Regional Development Fund (FEDER). R.H.B. acknowledges partial support from Universidad de La Serena Project DIULS CD08102. We are grateful for generous allocations of observing time at the Observatorio de Sierra Nevada and the Las Campanas Observatory. Thanks to the anonymous referee for some interesting suggestions that led to additional relevant information. The Space Telescope Science Institute is operated by the Association of Universities for Research in Astronomy, Inc., under NASA contract NAS5-26555.

\section{REFERENCES}

Abt, H. A., Levy, S. G., \& Gandet, T. L. 1972, AJ, 77, 138

Arias, J. I., Barbá, R. H., Gamen, R. C., et al. 2010, ApJ, 710, L30

Barbá, R. H., Gamen, R., Arias, J. I., et al. 2010, RevMexAA Conf. Ser., 38, 30 
Blaauw, A. 1993, in ASP Conf. Ser. 35, Massive Stars: Their Lives in the Interstellar Medium, ed. J. P. Cassinelli \& E. B. Churchwell (San Francisco, CA: ASP), 207

Bolton, C. T., \& Rogers, G. L. 1978, ApJ, 222, 234

Boyajian, T. S., Beaulieu, T. D., Gies, D. R., et al. 2005, ApJ, 621, 978

Crowther, P. A. 2007, ARA\&A, 45, 177

Feast, M. W., Thackeray, A. D., \& Wesselink, A. J. 1963, MmRAS, 68, 1

Fraser, M., Dufton, P. L., Hunter, I., \& Ryans, R. S. I. 2010, MNRAS, 404, 1306

Garrison, R. F., Hiltner, W. A., \& Schild, R. E. 1977, ApJS, 35, 111

Heger, A., \& Langer, N. 2000, ApJ, 544, 1016

Herrero, A., Kudritzki, R. P., Vilchez, J. M., et al. 1992, A\&A, 261, 209

Howarth, I. D. 2008, http://www.lowell.edu/workshops/Contifest/talks/Howarth. pdf

Howarth, I. D., Siebert, K. W., Hussain, G. A. J., \& Prinja, R. K. 1997, MNRAS, 284,265

Howarth, I. D., \& Smith, K. C. 2001, MNRAS, 327, 353

Kendall, T. R., Lennon, D. J., Brown, P. J. F., \& Dufton, P. L. 1995, A\&A, 298, 489

Langer, N. 1992, A\&A, 265, L17

Langer, N., Cantiello, M., Yoon, S.-C., et al. 2008, in IAU Symp. 250, Massive Stars as Cosmic Engines, ed. F. Bresolin, P. A. Crowther, \& J. Puls (Cambridge: Cambridge Univ. Press), 167

Lester, J. B. 1973, ApJ, 185, 253

Levato, H., Morrell, N., García, B., \& Malaroda, S. 1988, ApJS, 68, 319

Lyubimkov, L. S., Lambert, D. L., Korotin, S. A., et al. 2010, MNRAS, 410, 1774

Maeder, A., \& Conti, P. S. 1994, ARA\&A, 32, 227

Maeder, A., \& Meynet, G. 2000, ARA\&A, 38, 143

Mahy, L., Nazé, Y., Rauw, G., et al. 2009, A\&A, 502, 937

Maíz Apellániz, J., Sota, A., Walborn, N. R., et al. 2010, http://adsabs.harvard. edu/abs/2010arXiv1010.5680M

Marcolino, W. L. F., Bouret, J.-C., Martins, F., et al. 2009, A\&A, 498, 837

Martins, F., Schaerer, D., \& Hillier, D. J. 2005, A\&A, 436, 1049

Mathys, G. 1989, A\&AS, 81, 237
Meynet, G., \& Maeder, A. 2000, A\&A, 361, 101

Petrie, R. M., \& Pearce, J. A. 1961, Publ. Dom. Astrophys. Obs., 12, 1

Przybilla, N., Firnstein, M., Nieva, M. F., Meynet, G., \& Maeder, A. 2010, A\&A, 517, A38

Schaller, G., Schaerer, D., Meynet, G., \& Maeder, A. 1992, A\&AS, 96, 269

Schönberner, D., Herrero, A., Becker, S., et al. 1988, A\&A, 197, 209

Schönberner, D., Simon, K. P., \& Kudritzki, R. P. 1984, in Proc. Fourth European IUE Conf. (ESA SP-218; Noordwijk: ESA), 267

Simón-Díaz, S., Castro, N., García, M., Herrero, A., \& Markova, N. 2011, Bull. Soc. R. Sci. Liège, 80,514

Simón-Díaz, S., \& Herrero, A. 2007, A\&A, 468, 1063

Smith, K. C., \& Howarth, I. D. 1994, A\&A, 290, 868

Sota, A., Maíz Apellániz, J., Walborn, N. R., et al. 2011, ApJS, 193, 24

Villamariz, M. R., Herrero, A., Becker, S. R., \& Butler, K. 2002, A\&A, 388, 940

Walborn, N. R. 1970, ApJ, 161, L149

Walborn, N. R. 1971a, ApJ, 164, L67

Walborn, N. R. 1971b, ApJS, 23, 257

Walborn, N. R. 1973, AJ, 78, 1067

Walborn, N. R. 1976, ApJ, 205, 419

Walborn, N. R. 2003, in ASP Conf. Ser. 304, CNO in the Universe, ed. C. Charbonnel, D. Schaerer, \& G. Meynet (San Francisco, CA: ASP), 29

Walborn, N. R. 2009a, in Stellar Spectral Classification, ed. R. O. Gray \& C. Corbally (Princeton, NJ: Princeton Univ. Press), 66

Walborn, N. R. 2009b, in STScI Symp. Ser. 20, Massive Stars from Pop III and GRBs to the Milky Way, ed. M. Livio \& E. Villaver (Cambridge: Cambridge Univ. Press), 167

Walborn, N. R., Conti, P. S., \& Vreux, J.-M. 1980, PASP, 92, 284

Walborn, N. R., \& Fitzpatrick, E. L. 1990, PASP, 102, 379

Walborn, N. R., \& Howarth, I. D. 2000, PASP, 112, 1446

Walborn, N. R., \& Lennon, D. J. 2003, in IAU Symp. 215, Stellar Rotation, ed. A. Maeder \& P. Eenens (San Francisco, CA: ASP), 512

Walborn, N. R., Sota, A., Maíz Apellániz, J., et al. 2010, ApJ, 711, L143

Williams, S. J., Gies, D. R., Matson, R. A., \& Huang, W. 2009, ApJ, 696, L137

Wollaert, J. P. M., Lamers, H. J. G. L. M., \& de Jager, C. 1988, A\&A, 194, 197 